\title{
CORRELATION BEETWEEN SIZE-STRAIN POLE FIGURE AND TEXTURE
}

\author{
by TIDU Albert , HEIZMANN Jean-Julien \\ Laboratoire de Metallurgie Physique et Chimique \\ University of Metz
}

A complete line profile analysis consists of three stages :

- measurement of the line profile,

- corrections in view to obtain the only structurally broadened line profile,

- evaluation of structural parameters.

The measurement of line profiles is made by using a curved position sensitive detector (CPS 120) wich is able to register diffraction pattern on $120^{\circ}\left(2 \theta^{\circ}\right)$

We have two orders of line profile corrections :

- deconvolution : the observed line profile results of a convolution between the only structurally broadened line and the instrumental broadening,

- analytical corrections : background, Lorentz-Polarisation, absorption, smoothing of the line profile, ...

After these operations, we can obtain the structural parameters :

- the size of the coherently diffracting domains,

- the microstain arrising from small lattice distorsion and misfists in the cell structure (or the disorder coefficient).

The physically significance of these parameters is clear only for powder specimen. But the problem is quit difficult in case of bulk specimen. In all experiment, on cold work sample for example, one can see that the dislocation distribution, the faulting probability, the stored elastic energy are the major factors which regulate the domain size and the microstrain state. In case of material with residual stresses, the microstrain of unterest are those which are distributed around the mean strain value giving the macro-residual stress.

Normally, using a diffractometer with Bragg-Brentano focusing geometry, all the microstructural parameters found are related to the domains having a (hkl) plane parallel to the specimen surface. By using a $\psi$ goniometer with Bragg-Brentano focusing geometry, we can obtain these informations for all the sample position defined by the angles $(\phi, \psi)$ It is clear that these measurements are usefull only if the sample presents an anisotropy due to the texture. The case of the microstructure depending on the distance to the surface specimen is not traited here, consenquently we suppose that the texture is homogeneous in the 
diffracting volume. Therefore, the calculated values (size and micro-strain) are obtained for the diffracting domain at the $(\phi, \psi)$ position of the sample. By using all the calculated and measured values at the $(\phi, \psi)$ measurement directions we can construct size-strain pole figures on the same way as the common "integrated intensity" pole figure used for texture evaluation. We also can construct pole figure for all the line profile characteristics such as integral breadth, width at half maximum intensity, centroïde, form factor...

As it can be seen latter, these line profile characteristic pole figures are of great interest in material research because they give usefull informations about the structural anisotropy due to a given mechanical process. At the present time, the size-strain pole figure can only be used by comparaison to integrated intensity pole figure to obtain "visual" correlation, or analytical correlation such as relation between size-strain versus intensity.

\section{Size-strain separation:}

As it was seen in the introduction, the size-strain analysis cannot be performed on the measured line profile. An observed line profile results of the convolution between a standart profile $\mathrm{g}$ (wich describe the line broadening due to non-ideal geometry of the diffractometer or goniometer) and the only structurally broadenend profile $f(1)$ :

$$
h(x)=\int f(y) \cdot g(x-y) \cdot d y
$$

A deconvolution is necessary to obtain profile $f$ by measuring $h$ and $g$ profiles.

The most commonly method used for size-strain analysis is the Warren-Averbach method(2). It can be performed only if we can measure two or more orders of a (hkl) reflection. In this way, it is a multiple-line method. Applying Warren's theory, the separation of size and strain broadening is based on the order dependencies of both contributions by using Fourier coefficients of the pure profile $f$ after deconvolution.

Nevertheless, two orders of a reflection are not always available. In obtaining size and strain parameters, we must use single-line or integral breadth methods. The reader is reported to reference (3) in wich a good survey of these methods is given.

All these methods require additionnal asumptions as compared to multiple line methods. These asumptions naturally limits interpretation of the results, and, consequently they must be regarded with care. Nevertheless, if only relative variations of structural parameters are of interest, single line method are usefull tools.

The method used in this work is the initial slope method which use Fourier coefficient of the line profile (4).

The Fourier coefficients of the f line profile are directly obtain by using the Stockes deconvolution (5):

$\mathrm{f}, \mathrm{h}$ and $\mathrm{g}$.

$$
F(n)=H(n) / G(n) \text { where } F, H, G \text { are complex Fourier coefficients of profiles }
$$

According to Warren's theory, the $A(n)$ coefficient of the $f$ profile result from the product:

(1) $\quad A(n)=A(n)^{S} . A^{D}(n)$ where $A^{S}(n)$ represent the size coefficient and $A^{D}(n)$ the distorsion coefficient. (In the original Warren-Averbach method, the second term is de pendent of the order of the reflection). 
For $\mathrm{n}$ small, the size coefficients $\mathrm{A}^{\mathrm{S}}(\mathrm{n})$ can be written as :

$$
\text { (2) } \quad A^{S}(n)=1-\frac{n a}{D}
$$

where $a$ is the inverse of the period of the Fourier analysis.

The disorder coefficients are expressed as :

(3) $A^{D}(n)-1-2 \pi^{2} m^{2}\langle z(n) 2\rangle$ where $\langle z(n) 2\rangle=n^{2}\left\langle\varepsilon^{2}(n)\right\rangle$ is the reduced mean-square displacement, $m$ the order of the reflection, and $\left\langle\varepsilon^{2}(n)\right\rangle$ the micro-strain. For cold-worked metals it has be shown that (6):

$$
\text { (4) }\left\langle\varepsilon^{2}(\mathrm{n})\right\rangle=\mathrm{G}^{2} / \mathrm{n} \text { a }=\mathrm{C} / \mathrm{n}
$$

where $\mathrm{G}$ is a material constant for a particular reflection and $\mathrm{C}$ the disorder coefficient. The initial slope of a plot of $A(n)$ versus $n$ give the average coherence length $D$ or size domain. By assuming that the size coefficients are given by the exponential form:

$$
\text { (5) } A^{s}(n)=e^{-\frac{n a}{D}}
$$

the disorder constant is obtained for the particular value $\mathrm{na}=\mathrm{D} / 2$ :

$$
\text { (6) } \mathrm{C}=\left(1-\frac{\mathrm{A}(\mathrm{D} / 2)}{\mathrm{e}^{-1 / 2}}\right) \mathrm{a} / \pi^{2} \mathrm{~m}^{2} \mathrm{D}
$$

The micro-strain can be obtained by using simultaneously relations (3) and (4) :

(7) $A^{D}(D / 2)=1-\pi^{2} m^{2}\left\langle\varepsilon^{2}(D / 2)\right\rangle D^{2} / 2 d^{2}$ where $d$ is the interplanar spacing

\section{Construction of a size-strain pole figure:}

The size-strain separation allow us to measure the two line profiles $\mathrm{h}$ and $\mathrm{g}$ to obtain $f$. The $h$ (broadened) line profiles are measured for all the direction of measurement given by the angle $(\phi, \psi)$. The $g$ (standart) line profiles are measured for only one $\phi$ position (but $\psi$ varying), because the "instrumental" function remain the same by rotating arround the normal of the surface sample but change by tilting the sample.

For all the measurement directions, the results are stored and the size-strain separation is made after the data collection.

\section{Remarks:} tive process,

the line profile limits at high and low angle are found automatically by an itera -

- the numerical smoothing ensure us that the line profiles are unambiguously de fined (7) (we use a modified version of Fourier filter with automatic scaling of the filter coefficients).

- by using a curved position sensitive detector we measure two or more line profi les simultaneously and we can obtain size-strain pole figure for many reflections at the same time. But the results obtained for the reflection, which is not in Bragg-Brentano arrangement, are related to other domains tilted appart the surface sample. 
4) Results:

We present the results obtained for two samples of copper :

- CU RL : copper as received recristallised and cold-rolled (about $50 \%$ of reduction)

- CU NRL : copper as received and cold-rolled at the same state.

The standart specimen is a copper annelead sheet at $850^{\circ} \mathrm{C}$ with grain larger then $50 \mu$.

The figures $1 \mathrm{a}, \mathrm{b}$ and $2 \mathrm{a}, \mathrm{b}$ present, on the left, the size pole figure and, on the right, the "integrated intensity" pole figure. One can see clearly that for preferential texture directions, the size domain are high (and inversely). The dashed area on size pole figure represents the area where the line profiles cannot be analysed with accuracy.

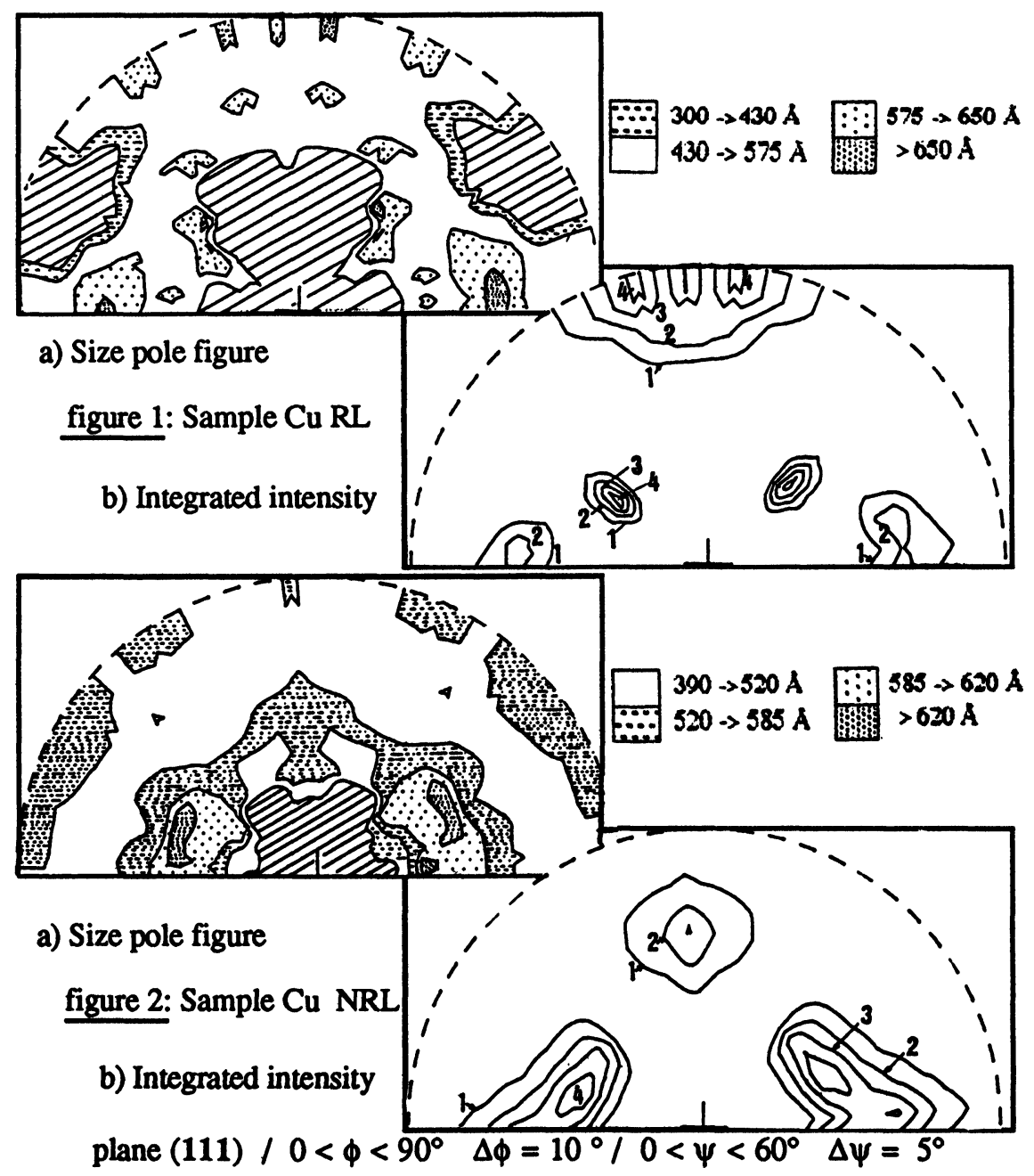


The mean size values for these directions are :

- CU RL : 700 Angstroem,

- CU NRL : 600 Angstroem.

By using the value obtained after size-strain separation, we have obtained other results (presented only for CU NRL).

As it can be seen on figure 3 a simple relation is obtained between size and microstrain at the value $\mathrm{L}=50 \AA$ (relation ( 7)):

$$
\left\langle\varepsilon_{50 \AA}>=K_{h k l} \cdot D^{-a_{h k l}} \text { with } a_{h k l}=0.91\right.
$$

$A$ analogeous relation is obtained between size and micro-strain at the value $\mathrm{D} / 2$ (or disorder coefficient). Previous works have shown that the constants $\mathrm{K}_{\mathrm{hkl}}$ and $\mathrm{a}_{\mathrm{hkl}}$ are order and sample dependent(8).

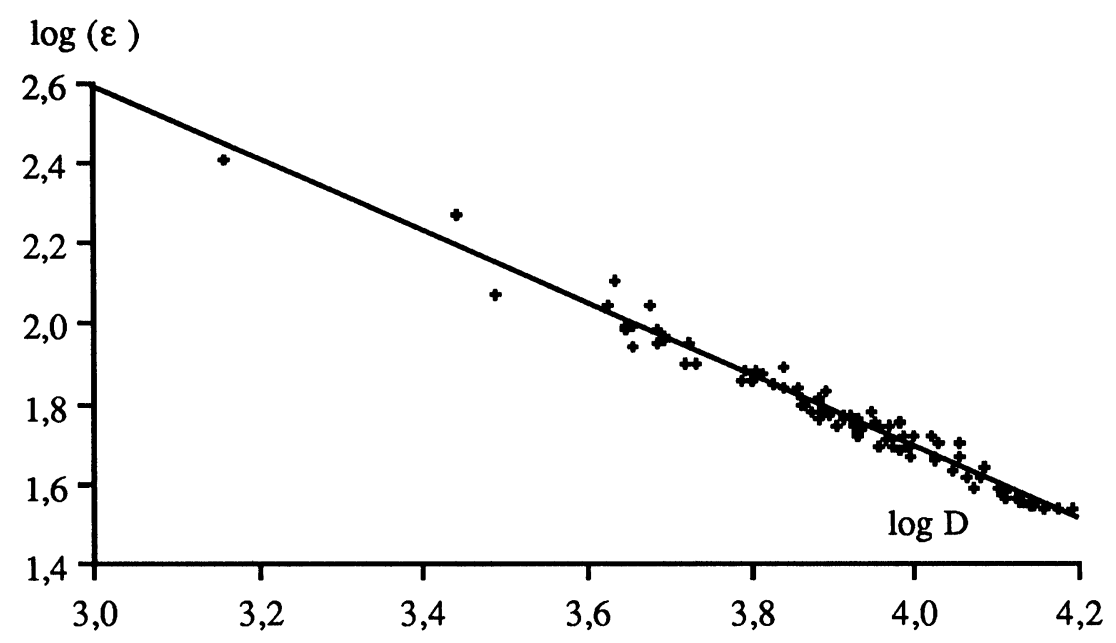

figure $3: \log (<\varepsilon 50 \AA>)=\mathrm{f}(\log \mathrm{D})$

The form factor $\varphi$ of the measured line profile defined by the ratio $2 \omega / \beta$ (full width at half maximum/integral breadth) is a good indicator of defect broadening rather the full width at half maximum. Qualitatively one can see, on unscaled figure 4, that for small values of $\varphi$, the strain broadening is small and size broadenening is high, and inversely for high value of $\varphi$. Figure 5 shows the variation of the form factor versus the integrated in tensity. It is clear that the integrated intensity slowly varies at high value of $\varphi$, and rapidly (with high fluctuation) at small value of $\varphi$. If we combine results of figures 4 and 5 we can see qualitatively that for direction of high intensity the strain broadening is high and the size broadening is low as it was been conclude from inspection of size pole figure. Similary results have been obtained for CU RL and for other samples such shot peened aluminium alloy and cold-rolled copper at $95 \%$ reduction. 

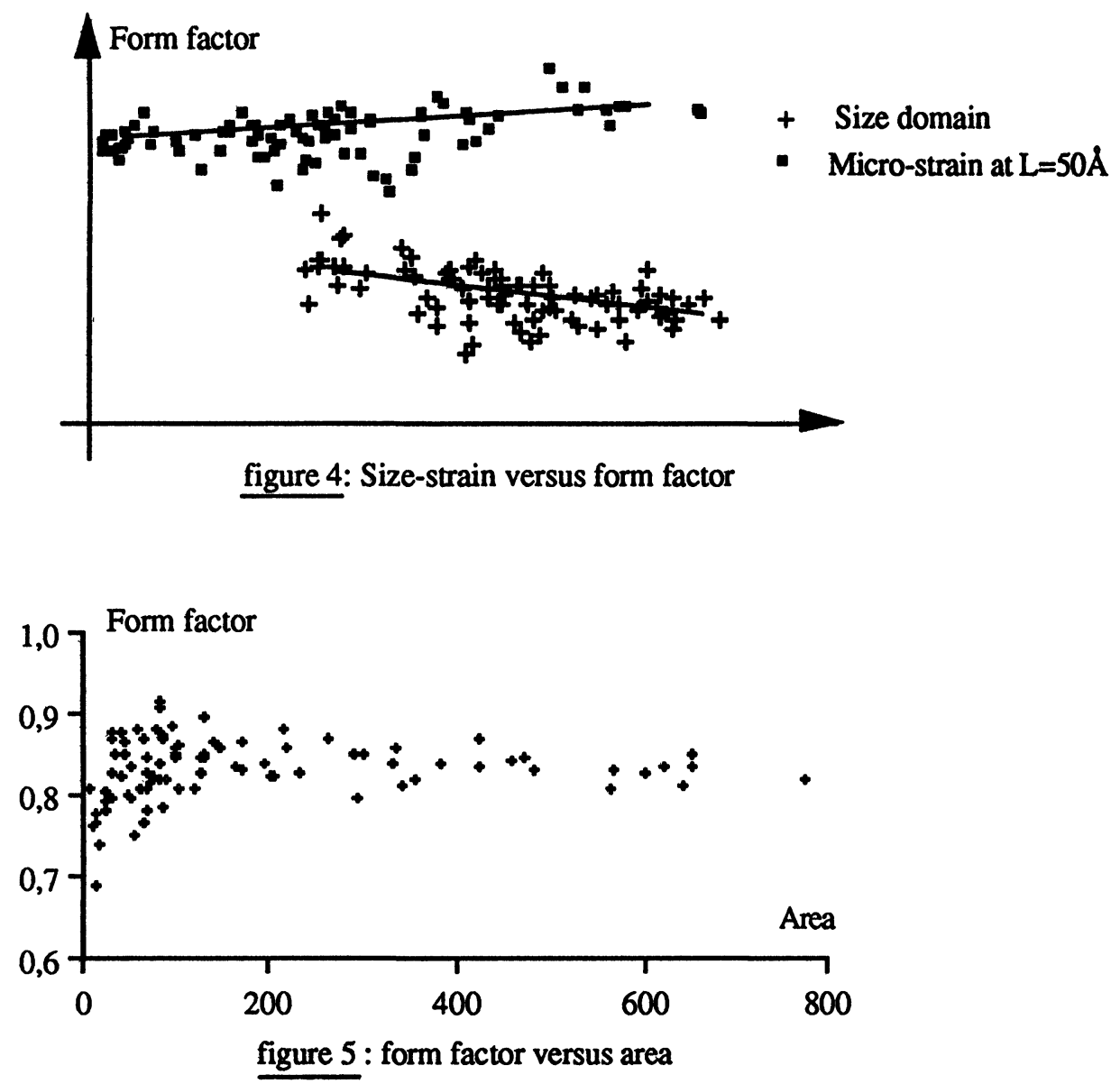

Bibligraphy:

1. JONES H.W., Proc. Roy. Soc., A166, 16, (1938)

2. WARREN B.E., AVERBACH B.L., J.Appl.Phys., 23, 497, (1952)

3. DELHEZ R., KEISJER Th. de, MITTEMEIJER E.J., Fresenius Z. Anal. Chem., 312, 1, (1982)

4. NANDI R.K., KUO H.K., SCHLOSBERG W., WISSLER G., COHEN J.B., CRIST

B. Jr., J. Appl. Cryst., 17, 22, (1984)

5. STOKES A.R., Proc. Phys. Soc. (London), B61, 382, (1948)

6. ROTHMAN R.L., COHEN J.B., J. Appl. Phys., 42, 971, (1971)

7. OLIVO O., Thesis University of Dijon, (1986)

8. TIDU A., Thesis University of Metz, (1990) 